\title{
In-situ time-resolved X-ray diffraction studies of crystalline materials under static mechanical load
}

\author{
V.I. Akkuratov ${ }^{1,2}$, A.V. Targonskiy ${ }^{1,2}$, A.E. Blagov ${ }^{1,2}$, Yu.V.Pisarevsky ${ }^{1,2}$, I.A. Eliovich ${ }^{1,2}$, A.I. Protsenko ${ }^{1,2}$, \\ M.V. Kovalchuk ${ }^{1,2}$ \\ ${ }^{1}$ FSRC "Crystallography and photonics" RAS \\ ${ }^{2}$ NRC "Kurchatov institute" \\ akkuratov.val@gmail.com
}

\begin{abstract}
A new approach to time-resolved X-Ray experiments implementation at both laboratory X-Ray sources and synchrotron facilities is presented. Proposed X-Ray diffraction method is based on adaptive X-ray optics and applied for investigation of irreversible deformation processes in crystalline materials under external loading with time resolution. This method allows receiving information about changes in atomic structure recording rocking curves (dependence of X-ray radiation intensity in the vicinity of Bragg angle) and reciprocal space maps (RSM) by fast tunable X-Ray optical element. This element consists of a piezoelectric monolithic bimorph lithium niobate $\left(\mathrm{LiNbO}_{3}\right)$ single crystal and a silicon plate attached to its face. When electrical signal is applied to the lithium niobate, it is possible to control the spatial position of the diffracted X-ray beam [1]. Time resolution of proposed method scales up to milliseconds for rocking curve record and up to hundreds of milliseconds in RSM case, and mainly depending on brilliance of X-ray.
\end{abstract}

The presented approach makes it possible to obtain information about changes in crystal structure with a lower time delay compared to existing methods [2].

The evolution of the defective structure of crystalline materials subjected to controllable and measured uniaxial mechanical compression (load up to $\mathrm{MPa}$ range) was investigated using the proposed method. The essence of such evolution is defect multiplication, displacement and shifting of atomic planes, which can be easily determined by changes in rocking curve and RSM parameters. This structural process is of great interest, as it is possible to observe defects behavior in a crystal during elastic deformation with time resolution.

The reported study was funded by the Russian Foundation for Basic Research grant №19-29-12037 mk and by Ministry of Science and Higher Education of the Russian Federation in the framework of the work on the state assignment of the Federal Research Center for Crystallography and Photonics, Russian Academy of Sciences.

[1] A. E. Blagov et al., Experimental Techniques, 517-523 (2017) 41 (5)

[2] Y. A. Eliovich et al., Crystallography Reports, 708-712 (2018) 63 (5)

Keywords: X-ray-optics, structure dynamics, Time-resolved X-ray studies 